\title{
Flow of foam through plain perforated and woven metal screens
}

\author{
Jacques T E Lemmen and Jan Groot Wassink
}

University of Twente, Enschede, The Netherlands

The interaction of foam with textile substrates has been investigated, starting with an attempt to describe the flow of foam in textile-like substrates. This was done by separately evaluating rheology and pressuredrop data. The influence of bubble size was found to be particularly important. In a coaxial cylinder viscometer the viscosity was found to be inversely proportional to bubble size. This finding could not be used to describe the pressure drop of foam flow through flat metal screens according to the d'Arcy equation. Equations could not be satisfactorily derived to describe phenomena such as bubble deformation and diminution, but empirical determination of these parameters could be achieved for each combination of foam and substrate.

\section{INTRODUCTION}

The use of foam in textile finishing has been the subject of increasing interest during of the 1980 s as a means of saving energy. This makes the study of the interaction between foam and substrate of some importance, particularly foam flow through and within the textile assembly. The problem of defining substrate geometry is one that must be tackled, and research has been done using air (permeability measurements) and Newtonian fluids that have resulted in extensive descriptions of the pressure-velocity relationship, which itself depends on geometrical parameters [1-4].

The study of foam flow has mainly been limited to "bulk foam flow' with its typical wall slip effects $[7,8]$ and to flow through porous media with considerable flow lengths. These investigations, reviewed by Heller et al. [5], have revealed the characteristic effects of compressibility and the importance of the ratio of bubble size to the "characteristic' size of the substrate [6]. The present investigation deals with the relationship between the foam rheology, as determined in a coaxial cylinder viscosity meter, and the flow behaviour through plain screens, with special attention being paid to the influence of mean bubble size.

\section{PERFORATED SCREENS}

\section{Experimental}

Pressure measurements on foam were all carried out using foam generated in a rotor-stator mixer. The foam solutions consisted of $10 \mathrm{~g} / \mathrm{A}$ sodium dodecyl sulphate in demineralised water. The foam was piped via a special measuring device through a $9 \mathrm{~m}$ long tube of less than 10 $\mathrm{mm}$ diameter. The purpose of this was to create a pressure in the mixer sufficient to insure that the mixing capacity was large enough to generate the amount of foam desired. The measuring device consisted of a tube with an almost oval opening at one end, in front of which the screen material was clamped and pressed against an O-ring. Openings of $1.24 \mathrm{~cm}^{2}$ and $2.99 \mathrm{~cm}^{2}$ were used and pressure was measured immediately outside the openings. Using the correlations found by Kroezen [6] a mean bubble diameter could be calculated to characterise the foam.

\section{Measurements on glycerol/water}

In order to determine a simple geometry dependence of the pressure-flow relationship all available mesh screens, ranging from mesh sizes from 40 to 215 (open area $7-23 \%)$ were measured. The results were evaluated as follows. Assuming laminar flow, a form of extended d'Arcy equation can be written (Eqn 1):

$$
\frac{v \eta}{\Delta p} \approx \frac{d^{2}}{L}
$$

The substrate can be regarded as a set of parallel pipes in which flow can be described by Poiseuille's law. Eqn 2 is the Hagen-Poiseuille equation in cylindrical pipes:

$$
\frac{v \eta}{\Delta p}=\frac{d^{2}}{32 L}
$$

Because there is little variation in $L$ (thickness of screens $84-118 \mu \mathrm{m})$, Eqn 3 is valid:

$$
\frac{v \eta}{\Delta p}=d^{a}
$$

where the exponent $a$ would be expected to lie between 1 and 2, and the fit with the experimental results to incorporate entrance effects. The resulting empirical relationship was found to be Eqn $4\left(\eta=0.09 \mathrm{~Pa} \mathrm{~s}\right.$ at $\left.22^{\circ} \mathrm{C}\right)$ :

$$
\frac{v \eta}{\Delta p}=0.67 d^{137}
$$

\section{Measurements on foam}

The first parameter to be investigated was the bubble size. Foams of constant density $(172 \mathrm{~g} / \mathrm{l})$ were generated at a constant flow rate $\left(10^{-4} \mathrm{~m}^{3} / \mathrm{s}\right)$ with a mean bubble diameter ranging from 45 to more than $350 \mu \mathrm{m}$. Screen hole diameters ranged from 42 to $312 \mu \mathrm{m}$. Some typical results can be seen in Figure 1, from which the pronounced influence of bubble size on the pressure can very clearly be seen. For mean bubble diameters less than $160 \mu \mathrm{m}$ the pressure seems to be inversely proportional to the mean 


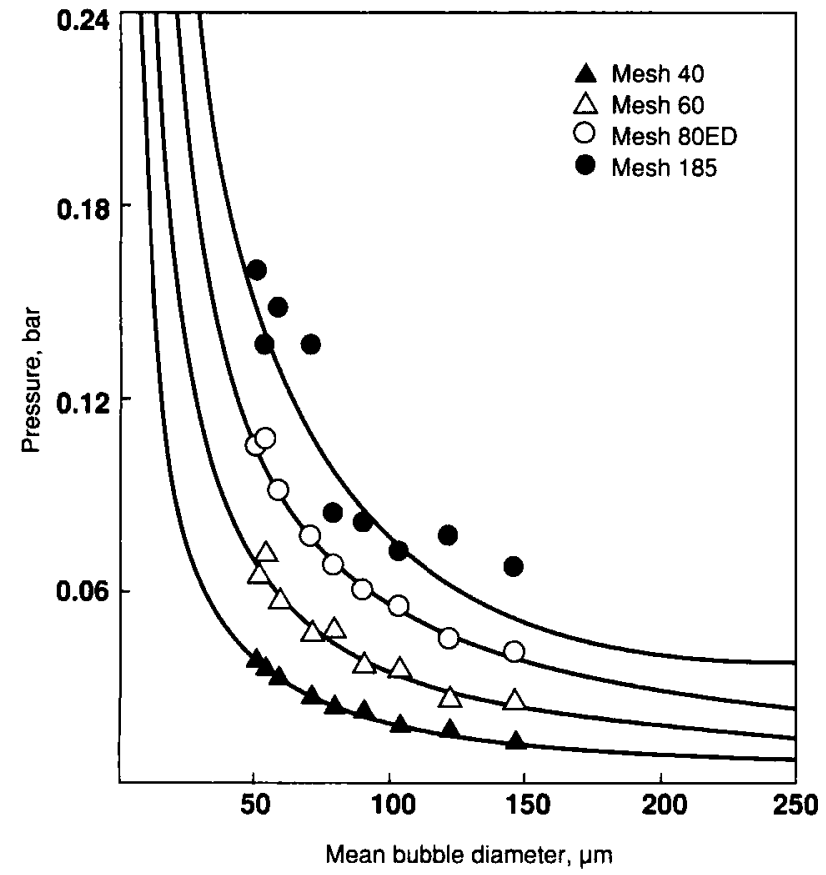

Figure 1 -Pressure of foam flow through screens

bubble diameter. One very remarkable feature that can be noticed is the very smooth path of the curves passing the point where the ratio $d_{b} / d_{h}$ changes from $<1$ to $>1$. This finding is similar to the relationship established by Hirasaki et al. [9] between apparent foam viscosity and the ratio $d_{\mathrm{b}} / d_{\mathrm{t}}$ (ranging from 0.1 to 30 ). A gradual increase in pressure level with increasing mesh number can be observed, due to a combination of the effects of hole diameter and actual open area.

The measured pressures cannot be used for evaluating for bubbles smaller than $160 \mu \mathrm{m}$ because in this region curves tend to cross each other. Therefore a mean pressure was calculated for bubbles larger than $180 \mu \mathrm{m}$ and an apparent viscosity for the foam using Eqn 4 was determined. Because no significant estimate of the velocity profile and resulting shear rates could be made, it was not possible to calculate a foam viscosity from known rheological constants. However, shear rate can be estimated from the $\bar{v} / d_{\mathrm{h}}$ ratio, and the way this relates to the apparent viscosity calculated from Eqn 4 can be assessed. This relationship is shown in Figure 2, which allows the following correlation to be drawn (Eqn 5):

$$
\eta_{\mathrm{a}} \approx\left(\bar{v} / d_{\mathrm{h}}\right)^{-0.54}
$$

which gives a value of 0.46 for the exponent in the power law, describing rheological behaviour by Eqn 6 :

$$
\eta_{\mathrm{a}}=\tau / D_{\mathrm{r}}=k\left(D_{\mathrm{r}}\right)^{n-1}
$$

The correlation (correlation coefficient $=0.97$ ) and the $n$ value are in good agreement, from which it may be concluded that, for this combination of foam and screen, a rheological approach combined with a d'Arcy type equation is valid, although this does not provide the exact numerical value of the constant $k$.

Because measurements were carried out in two flow areas, information was obtained at two superficial veloci-

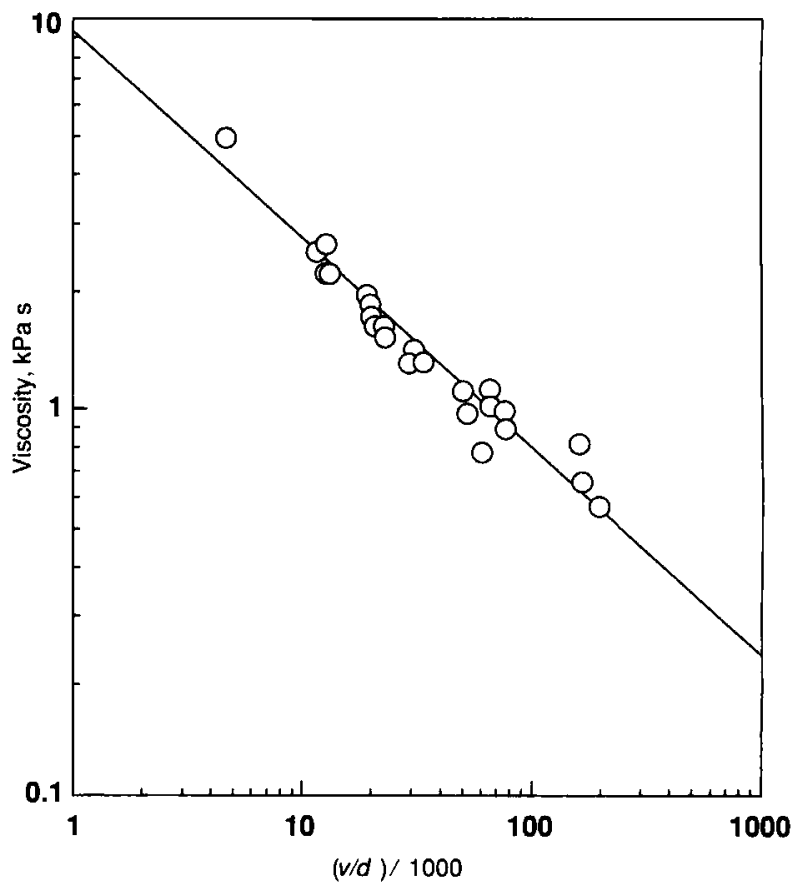

Figure 2 - Foam rheology for bubbles greater than $180 \mu \mathrm{m}$ diameter

ties. From this an $n$ value can be calculated according to the relationship $\Delta p \sim(\bar{v})^{n}$, which follows from combining Poiseuille's law with the power law for rheological behaviour (Table 1). The $n$ value is a mean value around which there is a great spread for the different mesh numbers $( \pm 30 \%)$. The value found for $d_{\mathrm{b}}>200 \mu \mathrm{m}$ can be compared to the 0.46 found for Eqn 4 . This gives a very clear tendency, i.e. an increase of $n$ when using smaller bubbles.

\section{TABLE 1}

\section{Values of $\boldsymbol{n}$ as a function of mean bubble diameter}

Mean bubble diameter $(\mu \mathrm{m})$ $n$ value

$\begin{array}{rr}59 & 0.69 \\ 122 & 0.61 \\ >200 & 0.50\end{array}$

Foam density $=172 \mathrm{~g} /$, foam flow $=1.0 \times 10^{-4} \mathrm{~m}^{3} / \mathrm{s}$, free flow area $=1.24 \alpha_{\circ}$ and $2.99 \alpha_{\circ} \mathrm{cm}^{2}$

The final interesting result on perforated screens was found by examining photographs from which the mean bubble diameter of foam having passed through the screen was determined. The mean bubble diameter of the foam entering the screen is marked on the $y$-axis of Figure 3, which shows that all the mesh screens caused the mean bubble diameter to decrease. Despite the large scatter of the results, a tendency for the mean bubble diameter to decrease more when using smaller screen meshes was observed. 


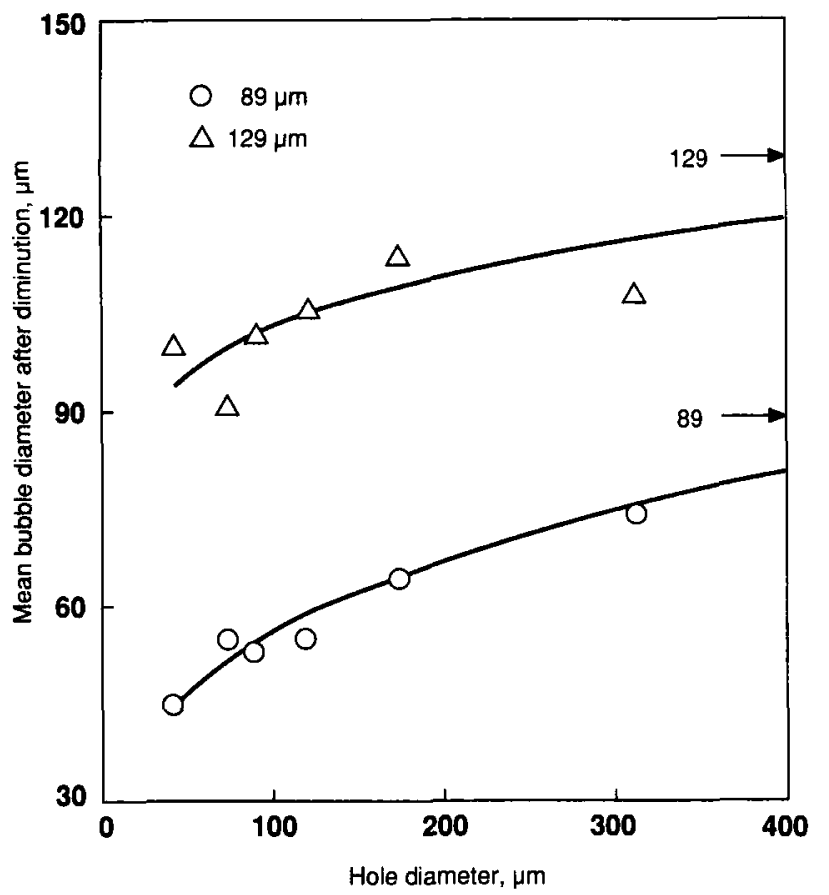

Figure 3-Diminution of bubble size through screens

It might be expected from this decrease in bubble size that an increase in viscosity would occur. Starting with a foam with a mean bubble diameter of $63 \mu \mathrm{m}$ and passing it through a woven screen (N4A) with holes of $66 \mu \mathrm{m}$ an increase in viscosity of 15-20\% was indeed found (Figure 4). Based on the viscosity of the foam entering the screen and the shear rate applied on the foam by the screen a mean bubble diameter of $40 \mu \mathrm{m}$ was calculated, using the theory of the critical Weber number [6]. These findings agree quite well with the results in Figure 3 (decrease of bubble size caused by mesh screens) and Table 2 (increase of viscosity at smaller bubble size).

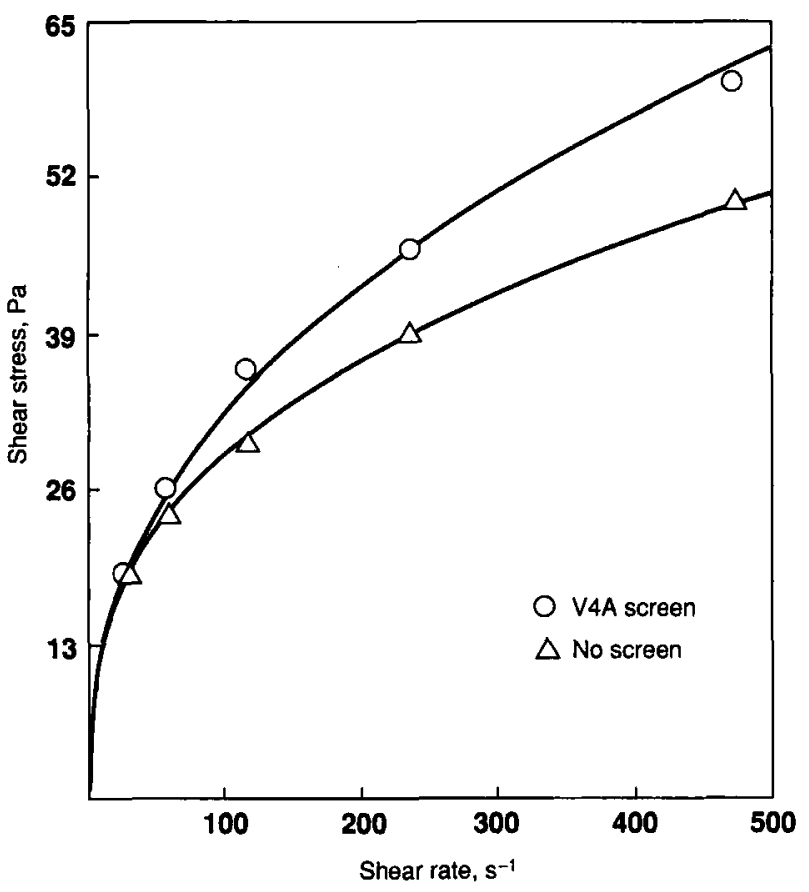

Figure 4 - Viscosity with and without a screen (V4A)
TABLE 2

\section{Values of $k$ as a function of mean bubble diameter}

Mean bubble diameter $(\mu \mathrm{m})$

$k$ value

$\begin{array}{rr}47 & 8.82 \\ 63 & 7.60 \\ 95 & 6.65 \\ 190 & 3.49\end{array}$

Foam density $=95 \mathrm{~g} / \mathrm{h}$, annular space $=1300 \mu \mathrm{m}$ (profiled) and $2000 \mu \mathrm{m}$ (smooth), shear rate $=20-1000 \mathrm{~s}^{-1}$

\section{WOVEN METAL SCREENS}

\section{Measurements on glycerol/water}

To find a workable relationship for the geometry dependence of the pressure-flow correlation Poiseuille's law was modified for a series of parallel, square pipes with a discharge coefficient found by other investigators $[10,11]$. It follows that Eqn 7 is applicable:

$$
\Delta p=\frac{8 \alpha \eta L \phi_{v}}{a^{4} \pi N}
$$

in which $\alpha$ is a constant expressing the geometric deviation of square and other non-cylindrical pipes from cylindrical pipes for which Poiseuille's law is valid.

Moreover, Eqn 8 was obtained from the literature $[10,11]$ :

$$
\Delta p=\frac{50 \eta\left(1-\alpha_{\mathrm{o}}\right) \phi_{\mathrm{v}}}{a^{3} N}
$$

and this enabled an expression for $\alpha$ to be written (Eqn 9):

$\alpha=\frac{50 \pi}{16} a\left(N_{\mathrm{a}}\right)^{1 / 2} \cdot\left\lfloor 2-d\left(N_{\mathrm{a}}\right)^{1 / 2}\right\rfloor\left(1+a^{2} N_{\mathrm{a}}\right)$

In the derivation of Eqn 9 a transformation from real perforated square holes in the screen to the woven structure was assumed, with $L=2 d$ (Appendix 1)

The measurements on three woven screens (Table 3) and at three different viscosities indicated a correction factor of about 0.56 to $\alpha$, attributed to the fact that the

TABLE 3

Geometry of woven screens (plain Dutch weave, steel)

\begin{tabular}{lcccc}
\hline Screen & $\begin{array}{c}\text { Holes } / \mathrm{m}^{2} \\
N_{\mathrm{a}}\end{array}$ & $\begin{array}{c}\text { Hole } \\
\text { diameter } \\
a(\mu \mathrm{m})\end{array}$ & $\begin{array}{c}\text { Wire } \\
\text { thickness } \\
d(\mu \mathrm{m})\end{array}$ & $\begin{array}{c}\text { Open } \\
\text { area } \\
\alpha_{\mathrm{o}}\end{array}$ \\
\hline Mesh-70 & $7.60 \times 10^{6}$ & 213 & 150 & 0.345 \\
Mesh-80 & $9.92 \times 10^{6}$ & 178 & 140 & 0.313 \\
V4a & $9.80 \times 10^{7}$ & 66 & 35 & 0.427
\end{tabular}


pipes were not square over the whole thickness of the screen but were rounded at both the entrance and the exit sides.

\section{Measurements on foam}

For $95 \mathrm{~g} / 1$ foams independent rheological and pressure measurements were carried out using woven screens. The measurements of shear stress versus shear rate were done point by point in a coaxial rheology meter. Both inner and outer cylinders were profiled and the space between them constantly filled with freshly generated foam with the desired mean bubble diameter. The desired shear rate was set while the foam flowed through the annular space. Measurements were taken by momentarily stopping the foam flow and reading the maximum shear stress before the foam degenerated and shear stress fell off rapidly.

From the curves in Figure 5 values for $n$ and $k$ for the different foams can be readily derived, $n$ being almost constant with a mean value of 0.38 while $k$ changed with bubble size (Table 2). For the region covered by Table 2 this means that $k$ was almost inversely proportional to $d_{b}$. For bubbles smaller than $47 \mu \mathrm{m}$ almost no increase in $k$ was found.

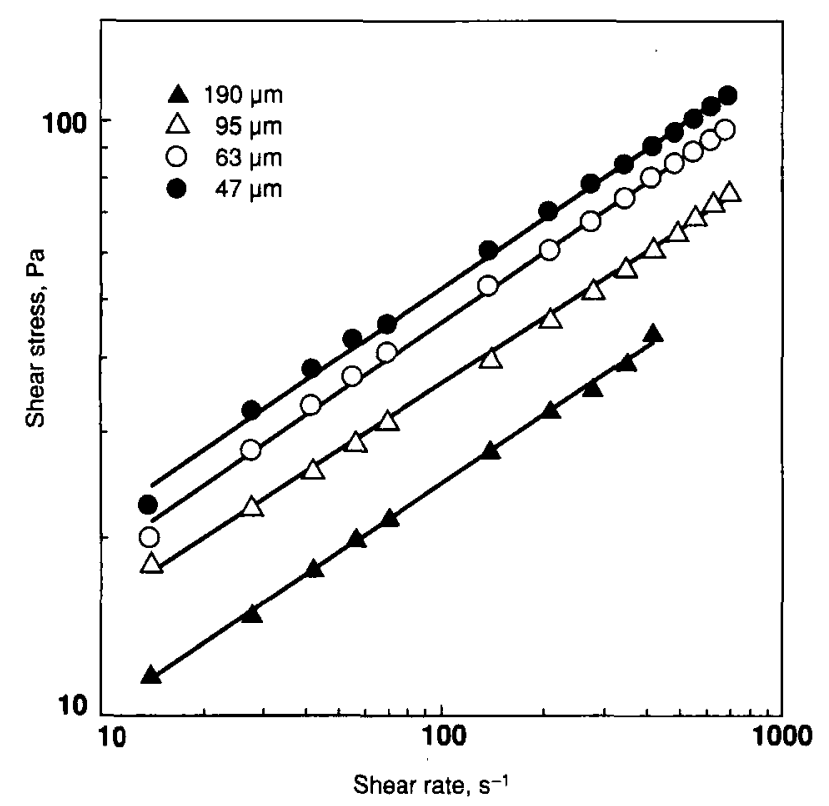

Figure 5 - Foam rheology (viscosity meter)

When pressures were measured across woven screens (Table 3), straight lines were again found when plotting $\Delta p$ against $\phi_{v}$ (e.g. Figure 6) but this time $n$ varied with mean bubble diameter as well as with the screen used (Table 4).

Assuming a model of parallel square pipes (Eqns 7-9) a value for $\Delta p$ can be calculated, using the rheological constants given in Table 2, $n=0.38$ and the correction factor extended for pseudoplastic behaviour. The measured pressure was found to be too low by a factor of about two when compared with the calculated value.

\section{EVALUATION}

An attempt was made to describe the flow of foam through substrates of plane geometry. Starting with Newtonian fluids, a very close correlation for perforated screens (Eqn 4) (correlation coefficient $=0.98$ ) and a reasonably good

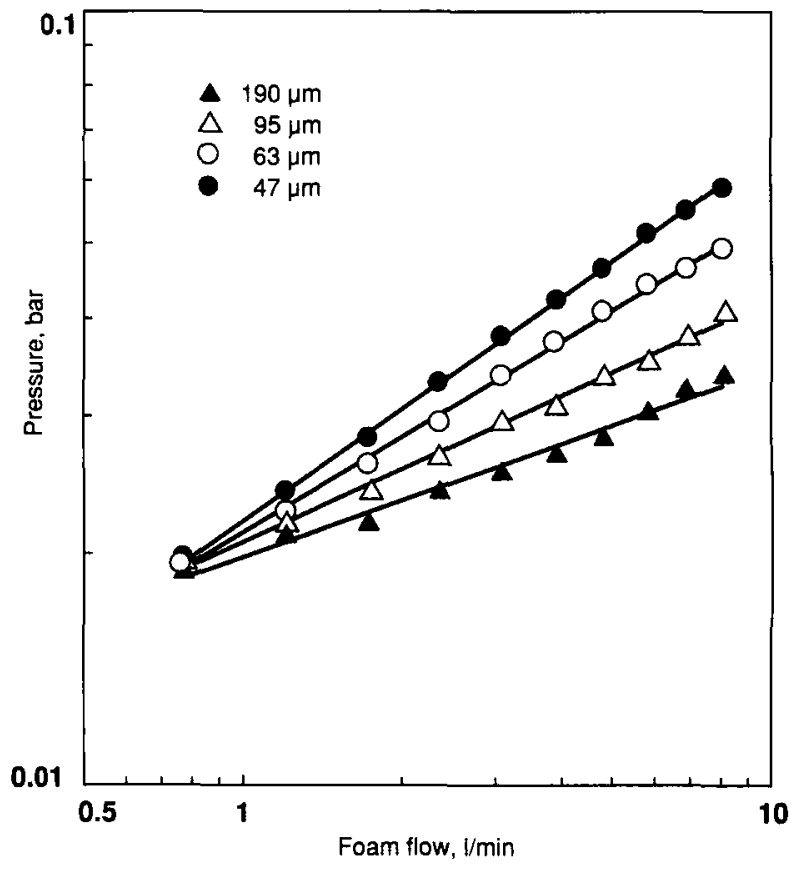

Figure 6 - Pressure of foam flow through a woven screen (V4A)

TABLE 4

Values of $\mathbf{n}$ as a function of mean bubble diameter and screen geometry (pressure)

$n$ values

\begin{tabular}{cccc}
\cline { 3 - 4 } $\begin{array}{c}\text { Mean bubble } \\
\text { diameter }(\mu \mathrm{m})\end{array}$ & Mesh-70 & Mesh-80 & V4A \\
\hline & & & \\
47 & 0.64 & 0.74 & 0.47 \\
63 & 0.57 & 0.63 & 0.41 \\
95 & 0.44 & 0.59 & 0.31 \\
190 & 0.42 & 0.48 & 0.24 \\
\hline
\end{tabular}

correlation for woven screens (Eqns 7-9) were established, being able to predict $\Delta p$ within $20 \%$. In both cases $\Delta p$ was found to be proportional to $\eta$ and to $v$ or $\phi_{v}$.

With these findings it was decided to combine the independently measured rheological properties with the equations obtained. This worked remarkably well for the combination of a coarse foam $\left(d_{b}>190 \mu \mathrm{m}\right)$, generally referred to as froth, with perforated mesh screens. Eqn 4 gave an $n$ value of 0.46 , which agreed very well with bulk foam rheology [6].

Substituting Eqn 9 into 7, with rheological properties following the power law equation $\tau=k\left(D_{\mathrm{r}}\right)^{n}$, gives Eqn 10 (Appendix 2):

$$
\Delta p=\frac{2.24 \alpha d k}{a^{3 n+1}} \cdot\left(\frac{3 n+1}{\pi n N_{\mathrm{a}} A}\right)^{n} \cdot \phi_{\mathrm{v}}^{n}
$$

However, it was impossible to obtain confirmation that this combined equation is universally applicable. Firstly $n$ 
varied with bubble diameter when determined from measurements of $\Delta p$ versus $v$ or $\phi_{v}$. The value of $n$ did remain constant for combinations with one or two smooth walls instead of two profiled cylinders (at lower shear rates deviations occur). Secondly $n$ was found to vary with geometry, which obviously is not consistent with it being a rheological constant. This means that Eqn 10 is applicable only to a combination of one mean bubble diameter with one type of screen. In this case $k$ and $n$ cannot be regarded as having any rheological significance.

Two further observations are relevant to an understand of the physical nature of foam flow through this kind of substrate: for perforated mesh screens $\Delta p$ was found to be inversely proportional to $d_{h}$ and $d_{b}$ to decrease considerably after the foam had passed through the screen.

Hence it was decided not to regard the foam as a continuum when flowing through plane porous substrates and therefore a foam bulk viscosity could no longer be used to describe the flow. Another aspect of geometry was therefore needed to try to understand its behaviour.

The viscosities of the separate gas and fluid phases in foam are too low to explain the pressure drop measured. Consideration of the bubbles themselves may provide an answer. According to Laplace's law the internal pressure of a bubble exceeds the pressure of its surroundings by $4 \gamma / d_{b}$. The pressures calculated from this are too low to account for the measured ones.

Other investigators report results that stress the importance of bubble size. Princen [12] has calculated a shear modulus (= shear stress/shear strain) that is inversely proportional to the bubble diameter (for $\tau<\tau$ ). Hirasakj et al. [9] found contributions from both bubble deformation and a surface tension gradient to the pressure of single bubbles in capilliaries. They used Poiseuille's equation to evaluate an apparent viscosity, found to be proportional to $1 / d_{b}{ }^{2}$ for a bulk foam and individual bubbles.

Although exact apparent viscosities could not be calculated, the dependence of bubble size can be evaluated using Eqn 11

$$
\eta_{\mathrm{a}}=C_{1} k\left(D_{\mathrm{r}}\right)^{n-1}=C_{2}\left(\bar{d}_{\mathrm{b}}\right)^{-m}
$$

and a value for $m$ for each level of shear rate $D_{\mathrm{r}}$ between 200 and $2000 \mathrm{~s}^{-1}$ was found using the results from Figure 6 and Table 5

The difference between the screens may be attributed to the effect of hole diameter (see Table 3). Even more

TABLE 5

Values of $\boldsymbol{m}$ at different shear rates (Eqn 11)

$m$ value at different shear rates

\begin{tabular}{lll}
\cline { 2 - 3 } Screen & $200 \mathrm{~s}^{-1}$ & $2000 \mathrm{~s}^{-1}$ \\
\hline V4A & 0.94 & 1.32 \\
Mesh-70 & 1.26 & 1.63 \\
Mesh-80 & 1.29 & 1.70 \\
\hline
\end{tabular}

important seems to be the effect of shear rate (velocity), which influences the dependence of bubble size. This effect is not incorporated into the model of Hirasaki et al., which is mainly concerned with slip flow over a liquid layer. In the present work 'structure' viscosity and bubble diminution seem to be the more important mechanisms.

When the bubble diminution mentioned in Figure 3 is taken into account, the extra pressure induced on bubbles when they are deformed or when they collapse in a shear field is apparent. According to Rumscheidt et al. [13], this extra pressure generated from inside the bubbles to compensate for the stress applied is inversely proportional with bubble size.

\section{CONCLUSION}

An attempt has been made to describe the flow of foam through plain metal substrates with the purpose of gaining an understanding of the mechanisms involved during the application of foam to textile (woven) structures, possibly through perforated mesh screens (as in printing).

It did not prove possible to describe the pressure drop found on the basis of a simple relationship such as d'Arcy's law and carefully measured, well defined bulk foam rheology.

The pressures were found to be too low to be explained by the bulk foam viscosity, and too high to be explained by bubble deformation or bubble splitting alone. The pressure-flow relationship was found to depend on bubble size and substrate geometry, but in a way that has not yet been explained. The substrate seems to act like a static mixer by diminishing the size of the bubbles when they pass through it.

This type of foam flow can best be described in terms of the combination effects of bulk foam viscosity and bubble distortion, because of the necessity to account for the contribution of foam structure. The 'structure' viscosity can be assumed to be inversely proportional to mean bubble diameter, an assumption that is consistent with theoretical calculations on dry foams (foam density approaching zero). For these foams Khan et al. [14] found the mean viscosity in steady shear flow to depend on $\left(D_{\mathrm{r}} a\right)^{-1}$, in which $a$ is a measure of bubble size. According to the theory of the critical Weber number $[6,13]$ for distortion and diminution of bubbles, the apparent viscosity would depend on $D_{\mathrm{r}}^{-1}$ and $d_{\mathrm{b}}{ }^{-1}$. The real shortcoming in all previous theoretical approaches emerges when the influence of the hole diameter through which the foam is forced comes to be considered. It must be concluded that pressure drop and apparent viscosity are largely determined by bubble size and are significantly influenced by hole diameter and shear rate.

In textiles processing Eqn 10 can be used for a combination of one substrate with one type of foam when the parameters $n$ and $k$ are experimentally determined. For the application on water-absorbing substrates the degradation of foam induced by capillary action would be expected to complicate the description of foam flow.

\section{REFERENCES}

\footnotetext{
1. W L Ingmanson et al., TAPPI, 44 (1961) 47.

2. J C Armour et al. Amer. Inst. Chem. Eng. J., 14 (1968) 415.

3. G C Pederson, Filtr. Sep., 11 (1974) 586.

4. A Rushton, Trans. Inst. Chem. Eng., 49 (1971) 49.

5. J P Heller et al. Ind. Eng. Chem. Res., 26 (1987) 318
} 
6. A B J Kroezen, PhD thesis, University of Twente (1988).

7. H G Wenzel et al., J. Materials, 5 (1970) 396.

8. J R Calvert et al., Int. J. Heat Fluid Flow, 7 (1986) 164.

9. G J Hirasaki and J B Lawson, Soc. Pet. Eng. J., 25 (1985) 186.

10. Perry's chemical engineers' handbook, 6th Edn 5-40

11. Grootenhuis, Proc. Inst. Mech. Eng. (London), A168 (1954) 837.

12. H M Princen, J. Coll. Interface. Sci., 91 (1983) 160

13. F D Rumscheidt, J. Coll. Sci., 16 (1961) 238

14. SA Khan and R C Armstrong, J. Non-Newtonion Fluid Mech., 22 (1986) 1

15. V G LeVich, Physicochemical hydrodynamics (New York: Prentice-Hall, 1962).

16. R B Bird et al., Transport phenomena (New York: John Wiley, 1960).

\section{LST OF SYMBOLS}

Symbol
$a$
$A$
$d$
$d$ (Eqn 9)
$d_{\mathrm{b}}$
$D_{\mathrm{r}}$
$k$
$L$


$n$
$N$
$N$
$N_{\mathrm{a}}$
$\Delta p$
$v$
$\bar{v}$
$\alpha$
$\alpha_{\circ}$
$\gamma$
$\eta$
$\eta_{\mathrm{a}}$
$\phi_{\mathrm{v}}$
$\tau$
$\tau_{\mathrm{o}}$

\section{Quantity}

Side of a square hole

Total flow area

Hole diameter

Thickness of a wire

Mean bubble diameter

Shear rate

Rheological constant

from the power law

Length or thickness in

the direction of flow

Rheological constant

from the power law

Number of holes

Number of holes $/ \mathrm{m}^{2}$

Pressure

Velocity in an aperture

Superficial velocity

Geometrical constant

Fraction of open area

Surface tension

Viscosity (Newtonian)

Viscosity at a certain

shear rate

Volume flow

Shear stress

Yield stress
Unit

$\mathrm{m}$

$\mathrm{m}^{2}$

$\mathrm{m}$

$\mathrm{m}$

$\mathrm{m}$

$\mathrm{s}^{-1}$

$\mathrm{Pa} \mathrm{s}^{-n}$

m

$\mathrm{m}^{-2}$

$\mathrm{Pa}$

$\mathrm{m} / \mathrm{s}$

$\mathrm{m} / \mathrm{s}$

-

$\mathrm{N} / \mathrm{m}$

Pa s

Pa s

$\mathrm{m}^{3} / \mathrm{s}$

$\mathrm{Pa}$

$\mathrm{Pa}$

\section{APPENDIX 1}

Eqn 9 was obtained by combining Eqns 7 and 8, making the following geometric assumptions:

$$
\begin{gathered}
\alpha_{\mathrm{o}}=N_{\mathrm{a}} a^{2} \\
1-\alpha_{\mathrm{o}}=2\left(N_{\mathrm{a}}\right)^{1 / 2} d-N_{\mathrm{a}} d^{2} \\
L=2 d
\end{gathered}
$$

When assessing the validity of the Poiseuille equation for application in the present study, the non-continuous character of the interaction between flow medium and substrate was completely neglected. The assumption of stationary flow was checked by evaluating a Fourier number that was analogous to the conditions stated by Levich [15] and by Bird et al. [16]. This condition states that

$$
N_{\mathrm{Fo}}=\frac{\eta t}{\rho d^{2}}>>1
$$

In general this condition will be satisfied by the very high viscosity and the low foam density.

\section{APPENDIX 2}

Eqn 10 was found by taking the basic differential equation for stationary (Appendix 1), laminar flow through circular tubes:

$$
\frac{\partial P}{\partial X}=-\frac{2 \tau}{r}
$$

and solving it for $\tau=k(\delta v / \delta r)^{n}$. This leads to the following expression for $\Delta p$ :

$$
\frac{\Delta p}{L}=\frac{2 k}{r^{3 n+1}}\left(\frac{3 n+1}{\pi n}\right)^{n} \phi_{v}^{n}
$$

which can be transformed to Eqn 10 by application to an array of parallel square pipes. 